\title{
Pollen as a target of environmental changes
}

\author{
Anil Grover ${ }^{1} \cdot$ David Twell $^{2} \cdot$ Enrico Schleiff $^{3,4,5}$
}

Published online: 10 June 2016

(C) Springer-Verlag Berlin Heidelberg 2016

Environmental alterations annotated as climate changes affect the activities and well-being of every organism. Ecosystems are influenced by longer-term, gradual alterations and by short-term sudden and severe weather phenomena, such as extreme temperature fluctuation, drought and heavy rainfall, resulting in some cases in migration or extinction. For humans the impact of climate change becomes more evident when agricultural production is disturbed by severe crop losses. This adds to our awareness of a global need for increased food production to support our expanding population, which in turn sets improvement of crop germplasm as one of the highest priorities for agriculturists, farmers, breeders and biologists. Reproduction is characterized as the developmental phase in plants most vulnerable to abiotic stresses, with particular sensitivity to increased temperature. Male gametophyte

Anil Grover

anil.anilgrover@gmail.com

$\triangle$ David Twell

twe@le.ac.uk

Enrico Schleiff

schleiff@bio.uni-frankfurt.de

1 Department of Plant Molecular Biology, University of Delhi South Campus, New Delhi 110021, India

2 Department of Genetics, University of Leicester, Leicester LE1 7RH, UK

3 Buchmann Institute for Molecular Life Sciences (BMLS), Goethe University, 60438 Frankfurt am Main, Germany

4 Cluster of Excellence Frankfurt, Goethe University, 60438 Frankfurt am Main, Germany

5 Department of Biosciences, Molecular Cell Biology of Plants, Goethe University, 60438 Frankfurt am Main, Germany development can be disturbed even under moderate temperature stress, a property shared by plants and mammals, but the basis for this susceptibility remains to be defined. This is challenging for plants, which have evolved complex stress response mechanisms, as a consequence of their sessile life style.

Current research seeks to identify the best germplasm for tailored breeding, to understand pollen development and fertilization and to characterize the reaction of the reproductive tissues to stressful conditions. These processes need to be described in detail and accordingly have attracted the attention of researchers in recent years. The reaction of developing pollen to stress conditions has been analyzed at different levels, combining a basic understanding of the global stress response systems in plants like Arabidopsis, tomato and rice, with high throughput technologies to decipher the global changes while coping with the stressful environment. The articles in this issue summarize current knowledge of male gametophyte stress responses at RNA, protein, lipid and epigenetic levels. Hafidh et al. (2016) provide an overview of cellular processes involved in pollen development and pollen tube growth including the mechanisms of communication between male and female gametophytes in the latter process and of stress response. A particular focus is placed on posttranscriptional regulation and the involvement of posttranslational modifications of proteins. This theme is transferred to the level of the regulation of the important processes by and of phospholipids in the following article by Heilmann and Ischebeck (2016). Particularly, phosphoinositides-which are the major focus of this articleemerge as new class of key regulators of pollen development and stress response, as well as of protein function. The understanding of the complexity of the latter required for pollen growth, adaptation and development is a key for 
a detailed description of the underlying processes. Thus, Chaturvedi et al. (2016) summarize the current advances in the description of the proteomic changes during development and stress adaptation, a review that guides the reader through the emerging wealth of information.

Chen et al. (2016) target a new emerging field, the impact of epigenetic events in male germ cell function and stress response. In the very recent years, theses mechanisms have attracted much attention, while an understanding of the regulatory regimes and the factors involved is just developing. Thus, the article combines both, a summary of the current knowledge and a discussion of the perspective in this field.

The significant advances made in describing the underlying principles of protein distribution networks, unfolded protein response and acclimation to high temperature are summarized in three complementary reviews. Paul et al. (2016) describes the pathways of protein distribution that have been described as they are central for the maintenance of pollen function during development or under stressful conditions. In addition to failure in distribution, it is described that proteins tend to aggregate under stressful conditions. Consequently, Fragkostefanakis et al. (2016) provide an overview on the mechanisms of unfolded protein response with focus on processes in pollen. Müller and Rieu (2016) broaden the view with respect to mechanism but focus on a particular threat by assigning the global regulatory regimes in pollen under heat stress.

Existing protocols for evaluation of pollen thermotolerance in main crops are also summarized and discussed. Mesihovic et al. (2016) provide guidelines for terminologies and experimental designs, which are of importance to unify information and to perform future experiments in a more standardized fashion, and Ambrosino et al. (2016) gives an overview at which places public available information can be found. Finally, what emerges is the need to integrate new large-scale datasets to extract key components of pollen vitality and to apply this knowledge to guide new breeding strategies, which is outlined in the article by Driedonks et al. (2016).

\section{References}

Ambrosino L, Bostan H, Ruggieri V, Chiusano ML (2016) Bioinformatics resources for pollen. Plant Reprod 29:133-147. doi:10. 1007/s00497-016-0284-8

Chaturvedi P, Ghatak A, Weckwerth W (2016) Pollen proteomics: from stress physiology to developmental priming. Plant Reprod 29:119-132. doi:10.1007/s00497-016-0283-9

Chen Y, Müller F, Rieu I, Winter P (2016) Epigenetic events in plant male germ cell heat stress responses. Plant Reprod 29:21-29. doi:10.1007/s00497-015-0271-5

Driedonks N, Rieu I, Vriezen WH (2016) Breeding for plant heat tolerance at vegetative and reproductive stages. Plant Reprod 29:67-79. doi:10.1007/s00497-016-0275-9

Fragkostefanakis S, Mesihovic A, Hu Y, Schleiff E (2016) Unfolded protein response in pollen development and heat stress tolerance. Plant Reprod 29:81-91. doi:10.1007/s00497-016-0276-8

Hafidh S, Fíla J, Honys D (2016) Male gametophyte development and function in angiosperms: a general concept. Plant Reprod 29:31-51. doi:10.1007/s00497-015-0272-4

Heilmann H, Ischebeck T (2016) Male functions and malfunctions: the impact of phosphoinositides on pollen development and pollen tube growth. Plant Reprod 29:3-20. doi:10.1007/s00497015-0270-6

Mesihovic A, Iannacone R, Firon N, Fragkostefanakis S (2016) Heat stress regimes for the investigation of pollen thermotolerance in crop plants. Plant Reprod 29:93-105. doi:10.1007/s00497-0160281-y

Müller F, Rieu I (2016) Acclimation to high temperature during pollen development. Plant Reprod 29:107-118. doi:10.1007/ s00497-016-0282-x

Paul P, Röth S, Schleiff E (2016) Importance of organellar proteins, protein translocation and vesicle transport routes for pollen development and function. Plant Reprod 29:53-65. doi:10.1007/ s00497-016-0274-x 